\title{
An Experimental Study of the Degradation of Particles in Complex Plasma
}

\author{
M. A. Ermolenko*, E. S. Dzlieva, V. Yu. Karasev, S. I. Pavlov, \\ V. A. Polishchuk, and A. P. Gorbenko \\ Fock Institute of Physics, St. Petersburg State University, St. Petersburg, 198504 Russia \\ *e-mail: plasmadust@yandex.ru \\ Received June 24, 2015
}

\begin{abstract}
Changes of melamine-formaldehyde resin (MF-R) particles occurring in complex dusty plasma have been investigated. Using a specially developed method, plasma-modified MF-R particles have been extracted from a dust trap after levitation for various periods of time and studied by electron microscopy techniques. Changes in the surface structure of MF-R particles are determined, and quantitative data on the variation in particle dimensions depending on the time of occurrence in plasma are obtained.
\end{abstract}

DOI: $10.1134 / \mathrm{S} 1063785015120196$

Investigations of complex plasma represent a rapidly developing field of interdisciplinary research, which is attractive in offering a rather broad range of possible applications both of plasma as such and plasma-modified dust particles [1,2]. The present Letter reports on the modification of polymeric melamine-formaldehyde resin (MF-R) particles during their occurrence in dusty plasma. Previous art includes the synthesis of particles in chemically active gases [3], plasma etching of particles with optical diagnostics of their dimensions [4], and studying the surface of particles treated in dusty plasma based on inert gases $[5,6]$. The latter became possible due to the development of methods for the extraction of particles from dusty plasma traps [7].

Experiments were performed in a discharge chamber equipped with an attachment for trapping, collecting, and extracting dust particles from glow discharge striations in the vertical part of the discharge tube. This experimental setup was previously used for selecting particles with respect to shape and size [79]. In the present study, we have used particles of melamine-formaldehyde resin (MF-R) with a density of $1.5 \mathrm{~g} / \mathrm{cm}^{3}$ and average initial diameter of $7.8 \mu \mathrm{m}$. Three-dimensional dusty plasma structures were created in several striations under selected levitation conditions: gas, neon; discharge current, $2.5 \mathrm{~mA}$; and pressure, 0.4 Torr. Under these conditions, a dust particle in plasma acquires a charge on the order of $10^{5}$ elementary units $(e)$ and can levitate in the vertical electric field of the dust trap. After a preset time, a collector device was placed under a striation from which dust particles dropped when the discharge was switched off. The deposited particles were collected using a mobile cartridge driven by a permanent magnet from outside the chamber. The cartridge carried a platform, on the surface of which the particles were collected. The platform was then transferred to and installed in the sample table of a scanning electron microscope (SEM). In every experiment, up to several thousand particles of dust were collected on the platform (Fig. 1a).

Using the above procedure, samples were collected in which the particles had been treated in plasma for 5 , 10,20 , and $25 \mathrm{~min}$ or not treated at all (initial particles kept in a separate container upon evacuation of the discharge chamber). These samples were examined by SEM (Merlin, Carl Zeiss). Figure 1b shows a typical image of a particle with a degraded surface.

The first discovered effect was a decrease in the average diameter of particles during their occurrence in the dust trap. The second effect was a change in the surface morphology of particles. Figure $1 \mathrm{~b}$ clearly reveals a developed surface relief with numerous "paths." Neither path length nor their mutual orientation on the surface revealed any regularity. However, the average path width increased with time of particle occurrence in plasma (Fig. 2).

Figure 3 presents a plot of average diameter $d$ of dust particles versus the time of levitation in plasma. The results showed that, under the experimental conditions indicated above, the volume of particles treated for $25 \mathrm{~min}$ decreased by up to $60 \%$ and the surface area increased by up to $45 \%$. Thus, a particle lost up to about half of its mass, while the size reduction led to a significant variation of charge during the experiment.

Technological processes of dry etching and surface treatment in plasma involve high-energy ions. In low- 

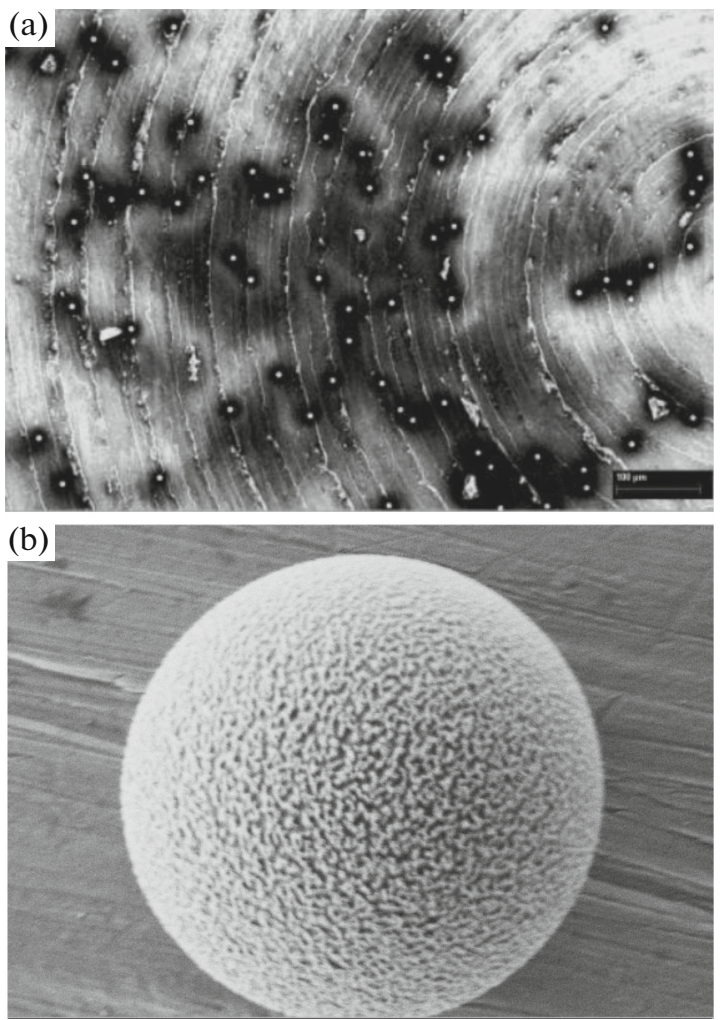

Fig. 1. SEM images of particles extracted from complex plasma: (a) MF-R particles collected on a substrate in one experiment (lateral image size, $0.8 \mathrm{~mm}$ ); (b) typical image of a modified spherical particle extracted from complex plasma, showing developed rough surface morphology (lateral image size, $10 \mu \mathrm{m}$ ). The time of particle occurrence in plasma was $20 \mathrm{~min}$.

temperature plasma of inert ions, the thermal energy of ions is on the order of $10^{-2} \mathrm{eV}$ and, hence, a question that naturally arises in considering the results of our experiments is what the mechanism of dust particle modification is. It can be suggested that ions incident onto the particle surface are accelerated by its field under conditions of charge maintenance. Under the experimental conditions, the average particle charge is on the order of $10^{5} \mathrm{e}$, while its (floating) potential is on the order of $10 \mathrm{~V}$. The energy of an ion reaching the particle surface is $\sim 6 \mathrm{eV}$ (i.e., significantly above the thermal energy). According to the equation of charging [1-3], the number of ions incident on the surface per unit time is on the order of $10^{3}$, with these ions imparting significant energy. The melting temperature of $\mathrm{MF}-\mathrm{R}$ is about $350^{\circ} \mathrm{C}$. Estimations show that the melted fraction of a particle during the observation time is comparable to the observed mass loss. Therefore, the degradation of particles in low-temperature complex plasma is probably caused by the ion flux incident on each particle under conditions of maintenance of its stationary charge.

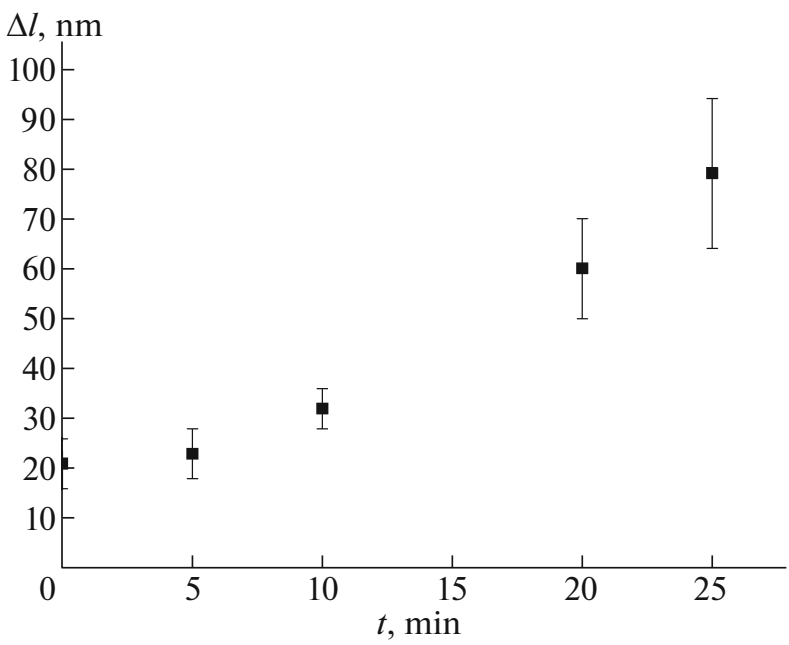

Fig. 2. Plot of average path width $\Delta l$ on the surface of dust particles vs. time of particle occurrence in the plasma dust trap (for experimental conditions, see text).

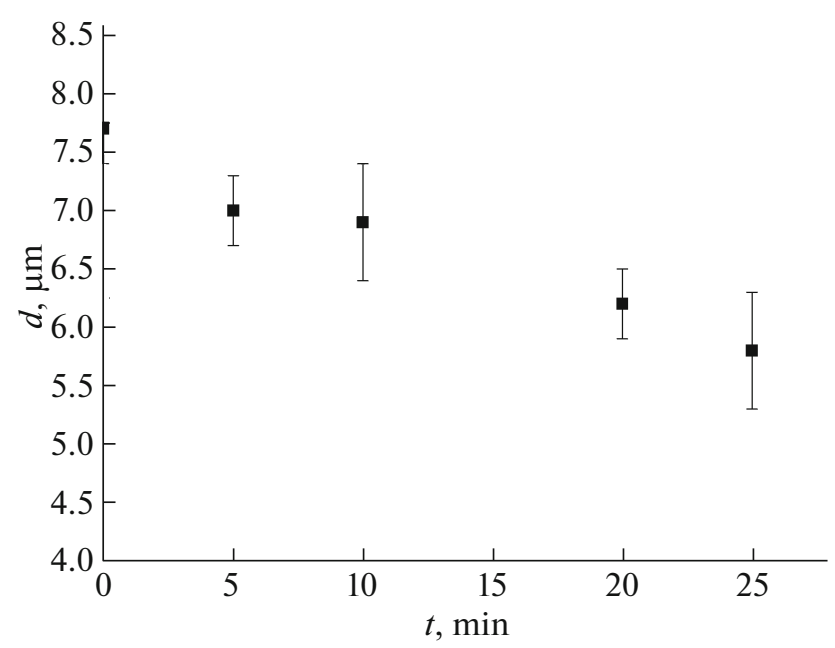

Fig. 3. Plot of average diameter $d$ of dust particles vs. time of particle occurrence in the plasma dust trap.

Thus, without drawing strict quantitative conclusions from the first experimental results, we can infer the following.

(i) In long-term experiments with complex plasma involving "soft" particles, it is necessary to allow for variation of the charge and mass of particles and take this into account in theoretical estimations.

(ii) Dusty plasma can be used for the processing of powders, e.g., for obtaining particles with developed porosity and roughness of the surface.

(iii) Dusty plasma can serve as a precise tool for obtaining particles of preset dimensions, e.g., those calibrated for optical diagnostics.

In conclusion, we have observed for the first time the degradation of dust particles during their occur- 
rence in complex plasma involving no chemically active gases.

Acknowledgments. This study was supported in part by the Russian Science Foundation, project no. 1412-00094.

\section{REFERENCES}

1. V. E. Fortov, A. G. Khrapak, S. A. Khrapak, V. I. Molotkov, and O. F. Petrov, Phys. Usp. 47, 447 (2004).

2. V. N. Tsytovich, G. E. Morfill, S. V. Vladimirov, and H. M. Thomas, Elementary Physics of Complex Plasmas (Springer, Berlin, 2008).

3. Dusty Plasmas: Physics, Chemistry, and Technological Impact in Plasma Processing, Ed. by A. Bouchoule (John Wiley \& Sons, New York, 1999).
4. W. W. Stoffels, E. Stoffels, G. H. P. M. Swinkels, M. Boufnichel, and G. M. W. Kroesen, Phys. Rev. E 59, 2302 (1999).

5. N. E. Zharikov et al., Uchen. Zap. Petrozavodsk. Gos. Univ. Estestv. Tekhn. Nauki, No. 6 (111), 99 (2010).

6. V. Yu. Karasev et al., Uchen. Zap. Petrozavodsk. Gos. Univ. Estestv. Tekhn. Nauki, No. 8 (145), 91 (2014).

7. V. Yu. Karasev, E. S. Dzlieva, A. Yu. Ivanov, and A. I. Eikhval'd, Phys. Rev. E 79, 026406 (2009).

8. E. S. Dzlieva, M. A. Ermolenko, and V. Yu. Karasev, Tech. Phys. 57 (7), 945 (2012).

9. E. S. Dzlieva, M. A. Ermolenko, and V. Yu. Karasev, Plasma Phys. Rep. 38 (7), 540 (2012).

Translated by P. Pozdeev 